\title{
From cirrhosis to paraparesis
}

\author{
Ioan-Cristian Lupescu', Speranta lacob ${ }^{2,4}$, Ioana Gabriela Lupescu ${ }^{3,4}$, \\ Liliana Gheorghe ${ }^{2,4}$, Adriana Octaviana Dulamea ${ }^{1,4}$ \\ ${ }^{1}$ Neurology Department, Fundeni Clinical Institute, Bucharest, Romania \\ ${ }^{2}$ Gastroenterology Department, Fundeni Clinical Institute, Bucharest, Romania \\ ${ }^{3}$ Radiology and Medical Imaging Department, Fundeni Clinical Institute, Bucharest, Romania \\ 4"Carol Davila" University of Medicine and Pharmacy, Bucharest, Romania
}

\begin{abstract}
We present the case of a 61-years-old male, diagnosed with liver cirrhosis, portal hypertension and type II diabetes mellitus, who developed bilateral signs of Parkinsonism, intention tremor and spastic paraparesis. Based on laboratory workup, brain CT, brain MRI and thoraco-lumbar MRI, a diagnosis of acquired hepato-cerebral degeneration with hepatic paraparesis was established. Although a rare entity, hepatic paraparesis needs to be considered in the right setting. As a result, we describe its key features, as well as new concepts that have emerged in recent years using advanced imaging, with emphasis on pathogenesis.
\end{abstract}

Keywords: hepatic myelopathy, globus pallidus, portosystemic shunt

\author{
Abbreviations (in alphabetical order) \\ CRP - C-Reactive Protein \\ CT - Computer Tomography \\ HE - Hepatic Encephalopathy \\ HM - Hepatic myelopathy \\ $\mathrm{HBV}$ - Hepatitis B virus \\ HDV - Hepatitis D virus \\ MELD - Model for End-stage Liver Disease \\ MEP - Motor evoked potentials \\ MRI - Magnetic Resonance Imaging \\ TIPS - Transjugular Intrahepatic Portosystemic Shunt
}

\section{INTRODUCTION}

Acquired hepato-cerebral degeneration is a rare irreversible neurologic syndrome occurring in patients with liver cirrhosis (1) after one or several episodes of hepatic coma (2). Its prevalence is not well known, however an analysis based on 1,000 cirrhotic patients found it to be $0.8 \%$ (3). Clinically, it manifests as a chronic progressive disorder comprising parkinsonism, ataxia, tremor, asterixis, choreoathetosis and cognitive disfunction $(2,4)$. A rare entity, termed hepatic paraplegia, is also known to complicate liver cirrhosis with portosystemic shunting $(2,5,6)$.

\section{CASE PRESENTATION}

We present the case of a 61 years-old male, diagnosed with liver cirrhosis of HBV+HDV etiology, with prophylactic esophageal banding and insulin-dependent type II diabetes mellitus. He came to our attention in February 2016, for neurologic assessment prior to inclusion on the liver transplantation waiting list. Apart from diminished ankle reflexes, neurologic exam was normal. Abdominal CT scan in March 2017 highlighted dilation of portal vein $(16 \mathrm{~mm})$, recanalized umbilical vein and gastric and splenic collateral circulation with splenomegaly. When he returned to us in May 2017, he 


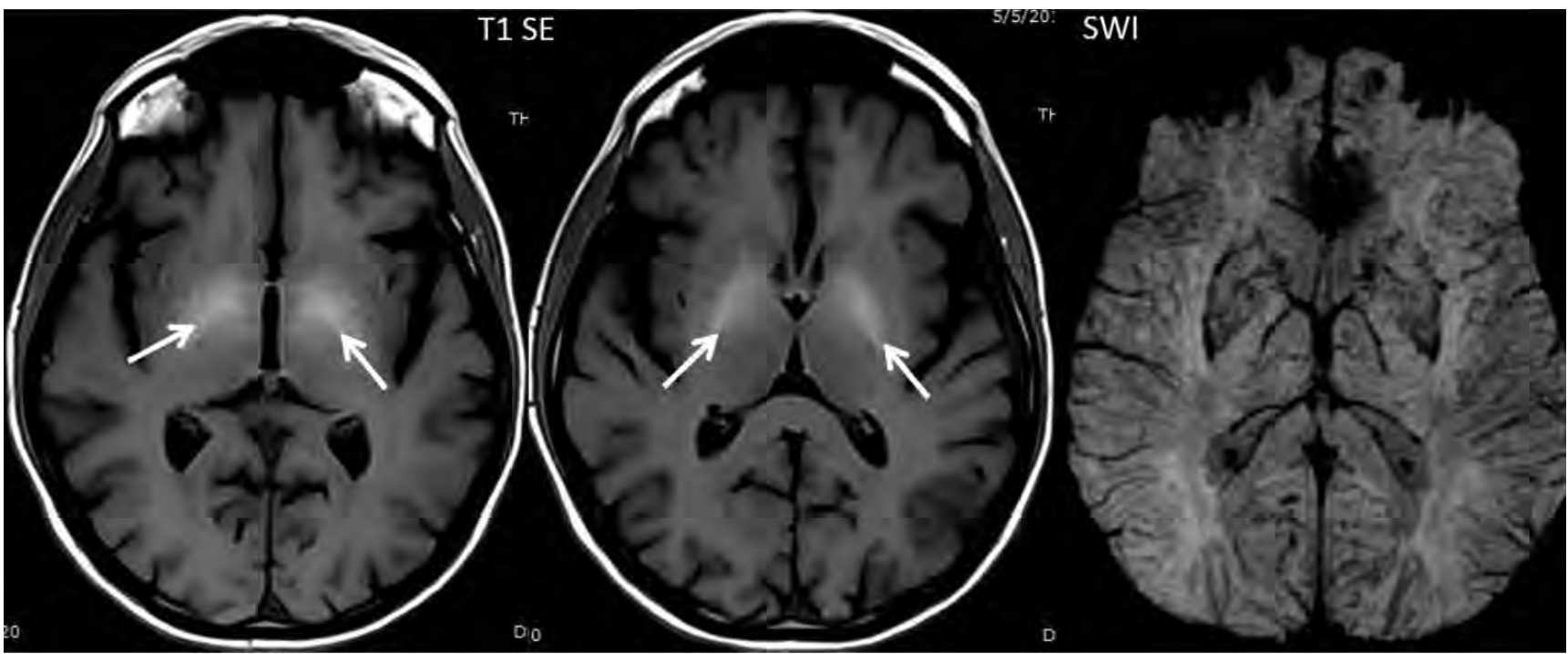

FIGURE 1. Axial T1 MRI: high signal intensities involving the globus pallidus bilaterally (related to manganese deposition).

exhibited bilateral cerebellar-parkinsonian tremor and cogwheel rigidity (both with predominant left involvement) and spastic paraparesis. Brain MRI was performed and showed characteristic bilateral T1 hyperintensities in the globus pallidus, with raised glutamate peaks on MR-Spectroscopy (Figure 1). Vitamin B12 deficiency was excluded, serum ammonia was slightly increased $(64 \mu \mathrm{g} / \mathrm{dl})$, and the severity of cirrhosis was calculated as a Child B9 with a MELD score of 22. Based on clinical and paraclinical examination, the patient was diagnosed with metabolic encephalopathy secondary to liver cirrhosis. Treatment included ammonia lowering drugs, namely Lactulose 1-2 measures q.d., Rifaximin $400 \mathrm{mg}$ t.i.d. and L-ornitine-L-aspartate b.i.d 14 days/month.

One month later, the patient developed sudden onset of fever and difficulty standing up. Clinical exam revealed fever $\left(38.1^{\circ} \mathrm{C}\right)$, left central facial palsy, ataxic tetraparesis with predominant left involvement and bilateral pyramidal syndrome. Brain CT was immediately performed, for suspicion of vertebrobasilar stroke, however, it did not show any acute lesions. Laboratory findings weren't significantly different from those of last admission, apart from hyperglycemia $(265 \mathrm{mg} / \mathrm{dl})$, slight leukocytosis $\left(11 \times 10^{3} / \mu \mathrm{l}\right)$ and hyperammonemia (110 $\mu \mathrm{g} / \mathrm{l})$. Blood and urinary cultures, tumoral markers and serological tests (for herpes simplex 1\&2, Epstein-Barr and syphilis) all came back negative and chest $\mathrm{x}$-ray was normal. The patient presented favorable evolution under Lactulose, Rifaximin, par- enteral amino acid solutions and adjustment of insulin therapy.

Thoraco-lumbar MRI was performed in July 2017 and excluded myelitis and compressive medullary causes for the patient's paraparesis. By that time, the patient could still walk, albeit unsteady and with a tendency for left-sided deviation. Unfortunately, because of advanced liver disease, the patient's clinical status worsened until exitus supervened.

\section{DISCUSSIONS}

In our case, the most probable cause for neurologic worsening was a new episode of hepatic encephalopathy (HE). It may not be uncommon for episodic HE to present with focal neurologic signs, as one prospective study over a 12-month period revealed a prevalence of $17.4 \%$ for such cases. These "atypical" presentations did not influence prognosis and were reversible, as no focal signs were identified at follow-up (7).

Our patient presented spastic paraparesis, which could not be attributed to a spinal cause, being most likely secondary to liver cirrhosis. This is known to occur, albeit rarely, hence the term hepatic myelopathy or hepatic paraparesis (HP). One thing to note is that HP has been reported with increasing frequency in the past few years. According to Conn et al, there were 61 cases described by 2006 (8), however a 2014 review on the subject noted approximately 90 cases reported in the literature (9). The usual clinical picture is that of a progressive spastic 
paraparesis, without sensory or sphincteric dysfunction (10). Actually, it is regarded as a rare complication of portosystemic shunting (11), not necessarily of liver dysfunction. This theory is backed up by several observations. For one thing, a temporal relationship exists between development of portosystemic shunting and HP. Wang et al noted progressive spastic paraparesis with lower limb hyperreflexia and Babinski signs in 4 out of 212 patients who underwent TIPS, with onset between 5 weeks and 5 months after TIPS creation. Spinal fluid analysis was within normal range and there were no signs of compression on imaging (12). In some cases, development of HP was reported a few years following spontaneous or therapeutic splenorenal shunting $(13,14)$. Due to portosystemic shunting, ammonia and (possibly) other intestinal metabolites bypass the liver, resulting in increased serum levels with consequent neurotoxic effects. Another observation compatible with this theory is that larger shunts have been associated with a more rapid onset of HP. For example, the median interval between construction of total portacaval anastomoses and development of HP was determined to be 16 months, however it was 60 months for partial non-portacaval shunts (8). Finally, spastic paraparesis can occur in the absence of liver failure, as was shown in one case of congenital hepatic fibrosis complicated by total portal-systemic shunting. All liver function parameters were within normal range, apart from elevated serum ammonia (15). Regarding clinical characteristics, one study compared 13 patients with HP with a control group of 487 subjects with chronic liver disease (without HP). All patients with HP were middle-aged (41-50 years-old) and there was a clear male-to-female predominance (12:1). Hypoproteinemia, blood ammonia levels and EEG abnormalities were found to have almost $100 \%$ negative predictive values and were regarded as significant diagnostic indicators for HP (16).

There is of course a long list of potential other causes that must be excluded before one established $\mathrm{HP}$ as the cause for paraparesis. Spinal cord MRI is usually negative and is therefore an essential tool to exclude compression and myelitis (8). In the setting of chronic liver disease, brain MRI shows typical T1 high signal intensities involving the globus pallidus (due to manganese deposition), coupled with raised glutamate and decreased myo-inositol and choline peaks on MR-Spectroscopy (attributed to brain hyperammonemia) (17). Globus pallidus T1 hyperintensities also occur in idiopathic portal hypertension and were shown to correlate with grade of portosystemic shunting, but not with severity of liver dysfunction (18). New information about the pathogenesis of HP emerged recently by using advanced imaging techniques. In one study with diffusion tensor imaging, cirrhotic patients with HP presented more changes of brain white matter tracts than cirrhotic patients without HP. In particular, alterations of the corpus callosum and superior longitudinal fasciculus were shown to correlate with motor deficits (19). Additionally, by using voxel-based morphometry, Liu et al demonstrated central gray matter abnormalities in liver failure patients with TIPS placement (compared with healthy controls). These were found predominantly in motor-related regions (thalami, basal ganglia and cerebellum). The authors noted a negative correlation between enlargement of the right caudate nucleus and lower limb motor performance, which might predict development of paraparesis (20).

Just as importantly, abnormal motor evoked potentials (MEP) have been demonstrated in all patients with HP $(6,21)$. Milder changes on MEP were also found in some patients with portosystemic shunting without clinical signs of HP, making them potentially useful for preclinical diagnosis (6).

Unfortunately, not much can be done to improve HP, once it becomes established. The condition does not usually respond to medical treatment (22), however it can be reversed in its early stages by liver transplantation (23). There have been reports of significant improvement following liver transplantation, including one in which the patient progressed from a wheelchair-bound state to full ambulation (24). A longer duration of HP seems to correlate inversely with clinical improvement after liver transplantation, hence it might be a negative prognostic factor for recovery (25).

\section{CONCLUSIONS}

Although considered rare, hepatic paraparesis has been encountered with increasing frequency in the past few years and should be known by both neurologists and gastroenterologists as a potential complication of liver cirrhosis. It remains however a diagnosis of exclusion. 


\section{REFERENCES}

1. J Lee, D Lacomis, S Comu, et al. Acquired hepatocerebral degeneration: MR and pathologic findings. Am J Neuroradiol. 1998; 19(3): 485-487.

2. Allan HR, Martin AS, Joshua PK. The Acquired Metabolic Disorders of the Nervous System. In: Allan HR, Martin AS, Joshua PK. Adams and Victor's Principles of Neurology. McGraw-Hill Educational, 2014: 1151-1152.

3. Fernández-Rodriguez R, Contreras A, De Villoria JG, et al. Acquired hepatocerebral degeneration: clinical characteristics and MRI findings. Eur J Neurol. 2010; 17(12): 1463-1470.

4. Meissner W, Tison F. Acquired hepatocerebral degeneration. Handb Clin Neurol. 2011; 100:193-197.

5. Mousseau R, Reynolds T. Hepatic paraplegia. Am J Gastroenterol. 1976; 66(4): 343-348.

6. R Nardone, Th Buratti, A Oliviero, et al. Corticospinal involvement in patients with a portosystemic shunt due to liver cirrhosis. J Neurol. 2006. 253: 81-85.

7. Cadranel JF, Lebiez E, Di Martino V, et al. Focal neurological signs in hepatic encephalopathy in cirrhotic patients: an underestimated entity? Am J Gastroenterol. 2001; 96(2): 515.

8. Conn HO, Rössle M, Levy L, et al. Portosystemic myelopathy: spastic paraparesis after portosystemic shunting. Scand J Gastroenterol. 2006; 41(5): 619-625.

9. R Nardone, Y Höller, M Storti, et al. Spinal cord involvement in patients with cirrhosis. World J Gastroenterol. 2014; 20(10): 2578-2585.

10. Koo JE, Lim YS, Myung SJ, et al. Hepatic myelopathy as a presenting neurological complication in patients with cirrhosis and spontaneous splenorenal shunt. Korean J Hepatol. 2008; 14(1):89-96.

11. Mendoza G, Marti-Fàbregas J, Kulisevsky J, et al. Hepatic myelopathy: A rare complication of portacaval shunt. Eur Neurol. 1994; 34(4): 209-212.

12. Wang MQ, Dake MD, Cui ZP, et al. Portal-systemic myelopathy after transjugular intrahepatic portosystemic shunt creation: report of four cases. J Vasc Interv Radiol. 2001; 12(7): 879-881.

13. Panicker J, Sinha S, Taly AB, et al. Hepatic myelopathy: A rare complication following extrahepatic portal vein occlusion and lienorenal shunt. Neurol India. 2006; 54(3): 298-300.
14. Yengue $P$, Adler $M$, Bouhdid $H$, et al. Hepatic myelopathy after splenorenal shunting: report of one case and review of the literature. Acta Gastroenterol Belg. 2001; 64(2): 231-233.

15. Demirci M, Tan E, Elibol B, et al. Spastic paraparesis associated with portal-systemic venous shunting due to congenital hepatic fibrosis. Neurology. 1992; 42(5): 983-985.

16. Yin YH, Ma ZJ, Guan YH, et al. Clinical features of hepatic myelopathy in patients with chronic liver disease. Postgrad Med J. 2009; 85: 64-68.

17. Rovira A, Alonso J, Cordoba J. MR imaging findings in hepatic encephalopathy. Am J Neuroradiol. 2008; 29(9): 1612-1621.

18. Fukuzawa $\mathrm{T}$, Matsutani $\mathrm{S}$, Maruyama $\mathrm{H}$, et al. Magnetic resonance images of the globus pallidus in patients with idiopathic portal hypertension: a quantitative analysis of the relationship between signal intensity and the grade of portosystemic shunt. J Gastroenterol Hepatol. 2006; 21(5): 902-907.

19. Wang LX, Guo L, Guo F, et al. Brain white matter fiber tracts involved in post-transjugular intrahepatic portosystemic shunt hepatic myelopathy. Neuroreport. 2017; 28(17): 1164-1169.

20. Liu K, Chen G, Ren SY, et al. Regional gray matter abnormality in hepatic myelopathy patients after transjugular intrahepatic portosystemic shunt: a voxel-based morphometry study. Neural Regen Res. 2019; 14: 850-857.

21. Nardone R, Orioli A, Höller $Y$, et al. Central motor and sensory conduction in patients with hepatic myelopathy. Spinal Cord. 2014; 52(6): 439-443.

22. Pinarbasi B, Kaymakoglu S, Matur Z, et al. Are acquired hepatocerebral degeneration and hepatic myelopathy reversible? J Clin Gastroenterol. 2009; 43(2): 176-181.

23. Rao PK, Sheth KA, Nadig R, et al. Portosystemic myelopathy: A rare neurological presentation of portosystemic shunts. J Clin Exp Hepatol. 2012; 2(4):393-395.

24. Caldwell C, Werdiger N, Jakab S, et al. Use of model for end-stage liver disease exception points for early liver transplantation and successful reversal of hepatic myelopathy with a review of the literature. Liver Transpl. 2010; 16(7): 818-826.

25. Qu B, Liu C, Guo L, et al. The role of liver transplantation in the treatment of hepatic myelopathy: Case report with review of the literature. Transplant Proc. 2009; 41(5): 1987-1989. 